EPJ Web of Conferences 64, 04002 (2014)

DOI: $10.1051 /$ epjconf/ 20146404002

(C) Owned by the authors, published by EDP Sciences, 2014

\title{
Accretion shock stability on a dynamically heated YSO atmosphere with radiative transfer
}

\author{
Lionel de Sá ${ }^{1,2, a}$, Jean-Pierre Chièze ${ }^{1, b}$, Chantal Stehlée ${ }^{2, c}$, Titos Matsakos ${ }^{1,2,3, d}$, Laurent Ibgui ${ }^{2, e}$, \\ Thierry Lanz ${ }^{4, f}$, and Ivan Hubeny ${ }^{5, g}$ \\ ${ }^{1}$ Laboratoire AIM, CEA/DSM - CNRS - Université Paris Diderot, IRFU/Service d'Astrophysique, CEA Saclay, \\ Orme des Merisiers, 91191 Gif-sur-Yvette, France \\ ${ }^{2}$ LERMA, Observatoire de Paris, Université Pierre et Marie Curie, ENS, Université de Cergy Pontoise and \\ CNRS, 5 Place J. Janssen, 92195 Meudon, France \\ ${ }^{3}$ CEA, IRAMIS, Service Photons, Atomes et Molécules, 91191 Gif-sur-Yvette, France \\ ${ }^{4}$ Laboratoire Lagrange, Université de Nice-Sophia Antipolis, CNRS, Observatoire de la Côte d'Azur, 06304 \\ Nice cedex 4, France \\ ${ }^{5}$ Steward Observatory, University of Arizona, 933 North Cherry Avenue, Tucson, AZ 85721
}

\begin{abstract}
Theory and simulations predict Quasi-Periodic Oscillations of shocks which develop in magnetically driven accretion funnels connecting the stellar disc to the photosphere of Young Stellar Objects (YSO). X-ray observations however do not show evidence of the expected periodicity.

We examine here, in a first attempt, the influence of radiative transfer on the evolution of material impinging on a dynamically heated stellar atmosphere, using the 1D ALE-RHD code ASTROLABE. The mechanical shock heating mechanism of the chromosphere only slightly perturbs the flow. We also show that, since the impacting flow, and especially the part which penetrates into the chromosphere, is not treated as a purely radiating transparent medium, a sufficiently efficient coupling between gas and radiation may affect or even suppress the oscillations of the shocked column.

This study shows the importance of the description of the radiation effects in the hydrodynamics and of the accuracy of the opacities for an adequate modeling.
\end{abstract}

\section{Accretion process in YSO}

Most Young Stellar Objects (YSOs) are surrounded by an accretion disk. Gas and dust follow a nearkeplerian infall from the outer disk down to a few stellar radii, the truncature radius, at which the magnetic pressure gets dominant over the thermal pressure of the gas. The flow is then magnetically confined in accretion columns [1]. High resolution observations [2] reveal the presence of a plasma

\footnotetext{
a e-mail: lionel.de-sa@cea.fr

be-mail: jean-pierre.chieze@cea.fr

ce-mail: chantal.stehle@obspm.fr

de-mail: titos.matsakos@cea.fr

e-mail: laurent.ibgui@obspm.fr

fe-mail: thierry.lanz@oca.eu

ge-mail: hubeny@as.arizona.edu
}

This is an Open Access article distributed under the terms of the Creative Commons Attribution License 2.0, which permits unrestricted use, distribution, and reproduction in any medium, provided the original work is properly cited. 
with typical coronal temperatures $\left(\sim 10^{6} \mathrm{~K}\right)$, but with higher electron density than in the coronal plasma $\left(10^{11}-10^{13} \mathrm{~cm}^{-3}\right.$ instead of $\left.\lesssim 10^{10} \mathrm{~cm}^{-3}\right)$. This feature is commonly interpreted as the spectral signature of shocks within an accretion funnel.

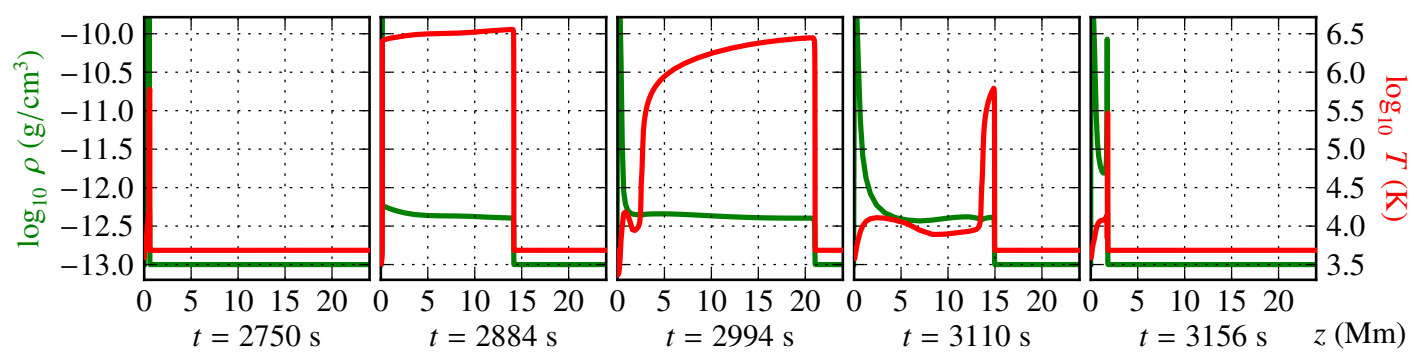

Figure 1. Snapshots of the density (green) and temperature (red) profiles of a QPO cycle ( $T \sim 400 \mathrm{~s})$; the accreted gas falls from the right at $v_{\text {acc }}=-400 \mathrm{~km} / \mathrm{s}$ on a "glass wall". From left to right: beginning of a new cycle $(t=2750 \mathrm{~s})$, growth of a hot slab of shocked material $(t=2884 \mathrm{~s})$, quasi-isochoric cooling at the slab basis (thermal instability, $t=2994 \mathrm{~s})$, collapse of the reverse shock $(t=3110 \mathrm{~s})$ and end of the collapse $(t=3156 \mathrm{~s})$. The escape probability of photons $\zeta$ is set to 1 (see section 3.2).

1D models [3-6] predict Quasi-Periodic Oscillations (QPO) of a shock in these columns: a first shock typically forms and stalls where the thermal pressure of the stellar atmosphere balances the ram pressure of the stream. A second shock (see Figure 1), the reverse shock, propagates back up the stream. The shocked gas, in between, cools down, resulting in the collapse of the whole structure. A new reverse shock forms then and a new cycle begins. All these models assume an optically thin coronal cooling (see section 3.2 for further details) and predict periods between 10 and $1000 \mathrm{~s}$. However, no such periodicity has been found in observations up to now [7, 8].

In order to understand the absence of QPO, the goal of this preliminary study is thus to include, for a first time in simulations of accretion flows, both radiative transfer and a model of a dynamically heated stellar atmosphere.

\section{Hydro-radiative simulations with the code ASTROLABE}

Computing accretion can be performed through two main approaches: on the one hand, 2D and 3D simulations can include the influence of the magnetic field [9] as well as geometric or flow inhomogeneities effects $[10,11]$; on the other hand, 1D simulations can hardly deal with these effects ${ }^{1}$, but may include more refined physics, like time-dependent (non-LTE) ionization or radiative transfer.

Our study is based on simulations performed with the 1D ALE code ASTROLABE [12] developed by one of us (J.-P. C.). This code solves the coupled equations of radiative hydrodynamics, written in the comoving frame. We consider here a system made of a plasma composed of $\mathrm{H}, \mathrm{H}^{+}, \mathrm{He}, \mathrm{He}^{+}$, $H e^{2+}, M$ and $M^{+}$, where $M$ represents a "catch-all metal" which completes, with the free electrons, the composition. The code is fully implicit, and of Adaptive-Lagrangian-Eulerian type: high resolution in regions of interest (e.g. large gradients) is achieved through a moving grid of fixed cardinality. In the following equations $\boldsymbol{u}$ and $\boldsymbol{u}_{r e l}$ denote the gas velocity and the relative velocity between the gas and the moving mesh points respectively.

Radiation is treated by solving for the two first moments of the radiation transfer equation (i.e. energy

\footnotetext{
${ }^{1}$ See e.g. [4] for an example of 1D simulations which include some effects of a slanted magnetic field.
} 
density $E_{r}$, and momentum density $\boldsymbol{M}_{\boldsymbol{r}}$ of the radiation field) [13, chapter 7] on the moving grid, but the radiative quantities are evaluated in the frame comoving with the gas. The resulting equations are:

$$
\left\{\begin{array}{l}
\frac{d}{d t} \int_{\mathcal{V}} E_{r} d \mathcal{V}+\int_{\mathcal{S}} E_{r} \boldsymbol{u}_{r e l} \cdot d \boldsymbol{S}+\int_{\mathcal{V}} \mathbb{P}_{r}: \boldsymbol{\nabla} \cdot \boldsymbol{u} d \mathcal{V}+\int_{\mathcal{S}} \boldsymbol{F}_{r} \cdot \boldsymbol{d} \boldsymbol{S}=\int_{\mathcal{V}} S_{E_{r}} d \mathcal{V} \\
\frac{d}{d t} \int_{\mathcal{V}} \boldsymbol{M}_{r} d \mathcal{V}+\int_{\mathcal{S}}\left(\boldsymbol{M}_{\boldsymbol{r}} \boldsymbol{u}_{r e l}\right) \cdot \boldsymbol{d} \boldsymbol{S}+\int_{\mathcal{V}} \boldsymbol{\nabla} \cdot \mathbb{P}_{\boldsymbol{r}} d \mathcal{V}+\int_{\mathcal{V}}\left(\boldsymbol{M}_{\boldsymbol{r}} \cdot \boldsymbol{\nabla}\right) \boldsymbol{u} d \mathcal{V}=\int_{\mathcal{V}} \boldsymbol{S}_{\boldsymbol{M}_{r}} d \mathcal{V}
\end{array}\right.
$$

where $\mathcal{S}$ denotes the surface of the control volume $\mathcal{V}$. The radiative energy and momentum source terms are respectively $S_{E_{r}}=\kappa_{p} \rho c\left(a T_{g}{ }^{4}-E_{r}\right)$ and $\boldsymbol{S}_{\boldsymbol{M}_{\boldsymbol{r}}}=-\kappa_{R} \rho c \boldsymbol{M}_{\boldsymbol{r}}$, where $\kappa_{P}$ and $\kappa_{R}$ are respectively the Planck and Rosseland mean opacities $\left(\mathrm{cm}^{2} \mathrm{~g}^{-1}\right)$. These terms realize the coupling between gas motions and radiation. These equations are completed by the M1 closure relation: $P_{r}=D E_{r}$ (in 1D), where the Eddington factor $0 \leq D \leq 1$, accounting for the anisotropy of the radiation, is calculated so as to maximize the local radiation field entropy $[14,15]$.

Ionization of the gas plays a key role in the pressure calculation and in the cooling of the gas. As an alternative to the usual treatment of ionization through a modified Saha equation $[3,16]$, we compute, coupled with hydrodynamics and radiative transfer equations, time-dependent coronal ionization using collisionnal ionization rates given by [17] and radiative recombination rates given by [18]. The ionization model also includes the hydrogen photoionization by the calculated ambient radiation field with local energy density $E_{r}(x, t)$.

\section{Shock oscillation perturbation}

Accretion shock observability depends both on their structure and on their burying in the stellar atmosphere. It is then important to model the structure on which accretion is performed, as well as to include radiation transfer to compute the radiative feedback on the column itself or on the surrounding atmosphere. In the following, we explore first the effect of the chromospheric dynamics on the accreted plasma, the later supposed to be optically thin. In a second step, we include the effect of the radiative transfer on the structure of the accretion flow.

\subsection{Chromospheric model}

Instead of using an ad hoc heating function to maintain the chromospheric equilibrium, we use a simple model which refers to the acoustic heating mechanism of the chromosphere of a star [1921]. We only aim here to get a self-consistent, fully hydro-radiative description of the chromospheric structure impacted by the accretion flow.

Figure 2. Formation and propagation of shocks induced by sound waves with an initial energy flux of $10^{8} \mathrm{erg} \cdot \mathrm{cm}^{-2} \cdot \mathrm{s}^{-1}$ and a period of $60 \mathrm{~s}$. The thin lines are successive snapshots of acoustic shocks in the chromosphere and the red thick line is the temperature profile of the solar chromosphere [22, and T. Lanz, priv. com.]. The escape probability of photons $\zeta$ is set to 0 (see section 3.2).

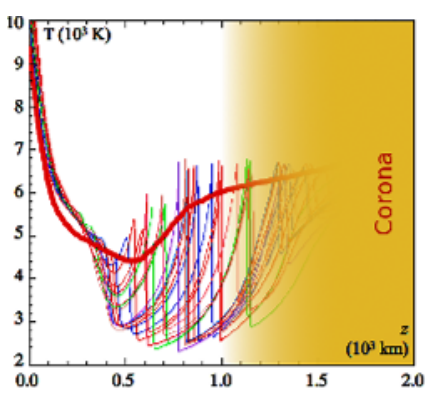


We have thus investigated the propagation of acoustic waves in the stellar atmosphere (we have chosen a Sun-like profile for fast qualitative comparisons with theoretical models and observations $[23,24])$. Mechanical energy is supplied at the base of the simulation domain, in the form of a monochromatic sinusoidal motion of the first (Lagrangian) interface in the simulation. Heating of the corona is not taken into account, since the later is readily crushed by the accretion flow. Figure 2 shows the formation of travelling shocks, induced by acoustic waves. Heating of the chromosphere is the result of the space- and time-averaged temperature structure above the photosphere.

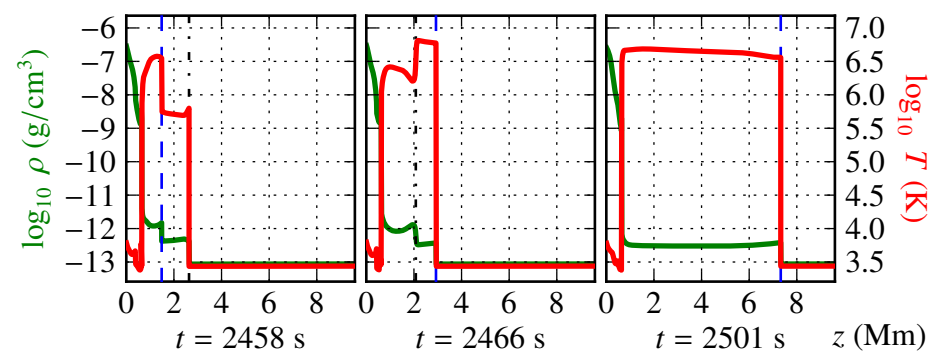

Figure 3. Snapshots of the density (green) and temperature (red) profiles of the end of a QPO cycle; gas fall at $v_{\text {acc }}=-400 \mathrm{~km} / \mathrm{s}$ on a dynamically heated chromosphere. From left to right: a new reverse shock (blue dashed line) is launched while the previous one (black dash-dot line) is still collapsing ( $t=2458 \mathrm{~s}$ ), the new reverse shock went beyond the old one $(t=2884 \mathrm{~s}$ ) and goes on propagating after the end of the collapse of the old reverse shock $(t=2501 \mathrm{~s})$. The escape probability of photons $\zeta$ is set to 0 in the atmosphere, and 1 in the accreted gas (see section 3.2).

To test the influence of this chromospheric model on the accretion structure, we compute accretion on either a fix, rigid, non-porous but transparent interface (a "glass wall", see Figure 1), or on a dynamically heated chromosphere (Figure 3). When acoustic shocks reach the hot slab, they are both reflected and transmitted. The transmitted wave hardly affects the shocked structure, apart from the end of the collapse: a new reverse shock (located by a blue dashed line in Figure 3 ) is created in the collapsing slab and come across the old one (black dash-dot line). At the end of the collapse, the newly formed hot slab is hardly sensitive to the chromospheric heating. The net result of the dynamical chromospheric heating is a cycle period slightly perturbed ( $\pm 50 \mathrm{~s}$ to the period, depending on the phase between the $60 \mathrm{~s}$ period acoustic heating and the $\sim 400 \mathrm{~s}$ period accretion cycle).

\subsection{Radiative transfer feedback}

The treatment of radiative transfer by the M1 method, adopted here, tackles the transition between the optically thick and thin regimes, using some set of Planck and Rosseland mean opacities calculated in the largest possible domain of physical conditions. However such calculation must generally rely on the assumption of LTE, which implies a common temperature for gas and radiation. However, this is not true at low densities, where coronal equilibrium prevails. The power radiated in coronal conditions is generally expressed as [25]:

$$
P=-n_{e} n_{H} \Lambda\left(T_{g}\right)
$$

while the equivalent (algebraic) energy sink/source term for the gas from the M1 method is:

$$
S_{E_{g}}=-\kappa_{p}\left(\rho, T_{g}\right) \rho c\left(a T_{g}^{4}-E_{r}\right)=-S_{E_{r}} \quad\left(\operatorname{erg} . \mathrm{cm}^{-3} \cdot \mathrm{s}^{-1}\right)
$$

The transition between these two regimes (which should be distinguished from the formal ability of the two moments M1 method to treat both optically thick and optically thin regimes) is a crucial 
issue regarding the treatment of the transition between the collision dominated plasma in the inner regions, and the non-LTE external region. As a first approach, we perform this transition by controlling the source and sink terms for matter and radiation energy by the escape probability of photons through the column $\zeta$; the effective energy source for the gas is then written as:

$$
S_{E_{g}}^{\mathrm{eff}}=\zeta P+(1-\zeta) S_{E_{g}}, \quad \zeta=1-\frac{1-e^{-3 \kappa_{P} \rho L}}{3 \kappa_{p} \rho L}
$$

where $L$ is a characteristic length scale of the order of the radius of the accretion column.

Figure 4. Snapshot of the density (green) and temperature (red) profiles of the accretion structure using a combined radiative cooling; gas fall at $v_{\text {acc }}=$ $-400 \mathrm{~km} / \mathrm{s}$ on a dynamically heated chromosphere. The hot slab height is reduced from $20 \mathrm{Mm}$ (Figures 1 and 3) to $2 \mathrm{~km}$.

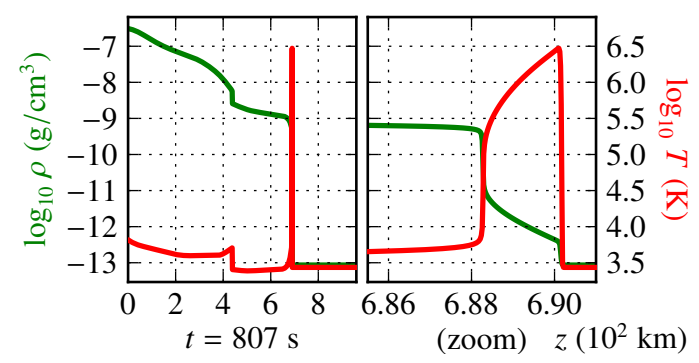

With combined opacities obtained from the Opacity Project [26-29] and Semenov tables [30], and adopting $L=1000 \mathrm{~km}$, the accretion shock gets stationary (Figure 4). Such structure is consistent with the non-periodic feature of the observations. However, the hot slab height (and the X-ray emitter volume, for a given column section) is reduced by a factor $10^{4}$ (from $20 \mathrm{Mm}$ down to $2 \mathrm{~km}$ ). The resulting small quantity of hot gas $\left(T_{g} \gtrsim 10^{6} \mathrm{~K}\right.$, see section 1$)$ cannot suffice to produce measured fluxes.

\section{Conclusion \& prospects}

We described in this paper a numerical model which includes for the first time the treatment of radiative transfer and a self consistent model of a stellar chromosphere, in order to precisely characterize the thermodynamical and radiative properties of the whole structure. The dynamical chromospheric heating slightly changes the predicted cycle period, whereas the cooling process strongly influences the shock structure and its observability.

Concerning the dynamics of the accretion flow, we have shown the importance of radiation transport, which relies on opacities. However, it is important to point out that the physical conditions of the plasma span a larger domain in $\rho$ and $T$ than the ones accessible in the two previous tables. Thus an ongoing improvement is to use new opacity tables from Ferguson [31, and Ferguson, priv. com.] and SYNSPEC [32]. Another one is to use more physical escape probabilities [33].

Radiation parameters in this work (energy, flux and opacities) are integrated over all frequencies. The underlying assumption, i.e. the spectral radiation energy distribution is a Planckian, is valid in the visible range, where photospheric emission dominates, but not in $\mathrm{X}$ rays, which include accretion shocks signature. An ongoing revision is to distinguish these radiation parameters in several frequency groups (visible+IR, UV, X) to take into account radiation specificities in each of them.

This work is supported by french ANR, under grant 08-BLAN-0263-07, Université Pierre et Marie Curie, Observatoire de Paris and CEA Saclay.

\section{References}

[1] J. Bouvier, S.H.P. Alencar, T.J. Harries, C.M. Johns-Krull, M.M. Romanova, Protostars and Planets V pp. 479-494 (2007) 
[2] B. Stelzer, J.H.M.M. Schmitt, Astronomy \& Astrophysics 418, 687 (2004)

[3] G.G. Sacco, C. Argiroffi, S. Orlando, A. Maggio, G. Peres, F. Reale, The Astrophysical Journal 491, L17 (2008)

[4] A.V. Koldoba, G.V. Ustyugova, M.M. Romanova, R.V.E. Lovelace, Monthly Notices of the Royal Astronomical Society 388, 357 (2008)

[5] G.G. Sacco, S. Orlando, C. Argiroffi, A. Maggio, G. Peres, F. Reale, R.L. Curran, Astronomy \& Astrophysics 522, A55 (2010)

[6] J.P. Chièze, L. de Sá, C. Stehlé, pp. 143-147 (2013)

[7] J.J. Drake, P.W. Ratzlaff, J.M. Laming, J. Raymond, The Astrophysical Journal 703, 1224 (2009)

[8] H.M. Günther, N. Lewandowska, M.P.G. Hundertmark, H. Steinle, J.H.M.M. Schmitt, D. Buckley, S. Crawford, D. O’Donoghue, P. Vaisanen, Astronomy \& Astrophysics 518, A54 (2010)

[9] S. Orlando, R. Bonito, C. Argiroffi, F. Reale, G. Peres, M. Miceli, T. Matsakos, C. Stehlé, L. Ibgui, L. de Sá et al., Astronomy \& Astrophysics (submitted) (2013)

[10] T. Matsakos, J.P. Chièze, C. Stehlé, M. González, L. Ibgui, L. de Sá, T. Lanz, S. Orlando, R. Bonito, C. Argiroffi et al., Astronomy \& Astrophysics 557, A69 (2013)

[11] T. Matsakos, EPJ - this conference (2013)

[12] P. Lesaffre, J.P. Chièze, S. Cabrit, G. Pineau des Forêts, Astronomy \& Astrophysics 427, 147 (2004)

[13] D. Mihalas, B.W. Mihalas, New York, Oxford University Press (1984)

[14] C.D. Levermore, Journal of Statistical Physics 83, 1021 (1996)

[15] B. Dubroca, J.L. Feugeas, Academie des Sciences Paris Comptes Rendus Serie Sciences Mathematiques 329, 915 (1999)

[16] J.C. Brown, Solar Physics 29, 421 (1973)

[17] G.S. Voronov, Atomic Data and Nuclear Data Tables 65, 1 (1997)

[18] D.A. Verner, G.J. Ferland, The Astrophysical Journal Supplement Series 103, 467 (1996)

[19] L. Biermann, Die Naturwissenschaften 33, 118 (1946)

[20] M. Carlsson, R.F. Stein, The Astrophysical Journal 397, L59 (1992)

[21] P. Ulmschneider, W. Rammacher, Z.E. Musielak, W. Kalkofen, The Astrophysical Journal 631, L155 (2005)

[22] J.E. Vernazza, E.H. Avrett, R. Loeser, The Astrophysical Journal 184, 605 (1973)

[23] W. Rammacher, P. Ulmschneider, Astronomy \& Astrophysics 253, 586 (1992)

[24] W. Kalkofen, The Astrophysical Journal 671, 2154 (2007)

[25] A.B. Kirienko, Astronomy Letters 19, 11 (1993)

[26] O.P. Team, The Observatory 116, 50 (1996)

[27] M.J. Seaton, Journal of Physics B: Atomic and Molecular Physics 20, 6363 (1987)

[28] N. Grevesse, A.J. Sauval, Space Science Reviews 85, 161 (1998)

[29] N.R. Badnell, M.A. Bautista, K. Butler, F. Delahaye, C. Mendoza, P. Palmeri, C.J. Zeippen, M.J. Seaton, Monthly Notices of the Royal Astronomical Society 360, 458 (2005)

[30] D. Semenov, T. Henning, C. Helling, M. Ilgner, E. Sedlmayr, Astronomy \& Astrophysics 410, $611(2003)$

[31] J.W. Ferguson, D.R. Alexander, F. Allard, T. Barman, J.G. Bodnarik, P.H. Hauschildt, A. Heffner Wong, A. Tamanai, The Astrophysical Journal 623, 585 (2005)

[32] I. Hubeny, T. Lanz, Astrophysics Source Code Library (2011)

[33] M. Carlsson, J. Leenaarts, Astronomy \& Astrophysics 539, A39 (2012) 